AN important control on the immune response in many animals, including rodents and primates, appears to be provided by the immune response (Ir) genes which are tightly linked to the major histocompatibility complex (MHC). These genes are antigen specific in their effects, that is, they appear to play an important part in determining whether an animal can respond to any particular antigen, but as yet their function and site of action is still unclear. However, evidence is rapidly accumulating that the Ir genes are in fact expressed in macrophages or the macrophage-like cells that process or present antigen to $\mathrm{T}$ lymphocytes.

Rosenthal and Shevach (J. exp. Med. 138, 1194, 1213; 1973) showed that guinea pig $T$ cells from an $F_{1}$ hybrid between a responder and a non-responder could only be stimulated to proliferate by antigen-coated responder (or $F_{1}$ ) macrophages, but not by non-responder macrophages. Subsequently, analogous proliferative studies have been performed in the mouse by Schwartz and his colleagues (J. Immun. 117, 531; 1976 and Proceedings 3rd Ir Gene Workshop, Asilomar, 1976). These studies suggested that the proliferating cells recognised antigen in association with macrophage Ir gene products, which since they were inhibited by anti-Ia antisera (antisera directed against the I region-associated antigens of the mouse MHC) were probably macrophage Ia antigens. While the immunological role of the proliferating cell(s) in these assays is not known, analogous results have been obtained in assays for the induction of antibody (Pierce et al. J. exp. Med. 144, 371; 1976) or of helper cells (the T cells which collaborate with B cells to enable the latter to produce antibody). For example, Erb et al. (Eur. J. Immun. 6, 365, 1976) have demonstrated the capacity of macrophage Ia

Marc Feldmann is an ICRF research fellow working at University College London.

that pulsations have been induced artificially near a pulsating display is unexpected and might give valuable insight into the mechanism causing the natural phenomenon.

The artificial pulsations were found by Deehr and Romick (this issue of Nature, page 135) in one of the natural auroral spectral lines at $200 \mathrm{~km}$ altitude, just below a barium release in the $F$ region of the ionosphere. This technique, in which $1.5 \mathrm{~kg}$ of $\mathrm{Ba}$ is ejected by a thermite reaction from a canister carried aloft by a rocket, is used to measure electric fields and

\section{Are the Ir genes expressed by macrophages?}

\author{
from Marc Feldmann
}

antigen, complexed to a fragment of immunogen, to form a soluble complex of molecular weight $\sim 55,000$ daltons, which replaces intact macrophages in the induction of helper cells. All these studies reinforce the concept that there are Ir gene effects expressed in macrophages. J. E. A. P. Miller and his colleagues have recently obtained compatible results in a delayed hypersensitivity (DTH) model in the mouse, leading to the same conclusion for the Ir genes involved in DTH (Miller et al. J. exp. Med., in the press).

In this issue of Nature, Rosenthal, Barcinski and Blake (page 156) extend this point. Using insulin molecules from various species, with minor differences in amino acid sequence, they demonstrate Ir gene control of antigen recognition at the level of the macrophage, measured by the proliferative response of guinea pig $\mathrm{T}$ cells. Their results are most easily interpreted by postulating that macrophages may select the appropriate portion of the antigen to be presented to $\mathrm{T}$ cells. How is this done? The simplest possibility is that macrophage Ir genes define a class of receptors of broad specificity that 'focus' or orient antigen for $T$ cells to recognise it. Conceivably, these receptors may be the descendants of the recognition molecules which antedate the vertebrate immune system. If that interpretation is correct the antigen-presenting cells, and not the $T$ cells, dictate the antigen specificity of the Ir gene effect. But since T cell responses depend so intimately on macrophage presentation, macrophage Ir genes would reveal them-

winds in the upper atmosphere. The barium cloud is partially ionised by sunlight, forming a cloud of ions which resonantly scatters purple light from the Sun and a neutral cloud which scatters green light. The neutral cloud is blown across the sky by winds while the ionised cloud is driven by the combination of electric fields and winds. By tracking both clouds optically from the ground the effects of the two forces can be separated. The technique is very effective and has been used many times in the last decade.

Ideally the barium should not dis- selves (as has been found) as defects of $\mathrm{T}$ cell function. The search for the immune response gene products may then already be partly over-they have been isolated and partly purified as the macrophage Ia antigenimmunogen complex, which induces the activation of helper cells in vitro.

The idea that the Ir genes are expressed on macrophages is also compatible with other findings that have been interpreted quite differently. Munro and Taussig for example (Nature 256, 103; 1975), described two types of mice with genetically determined low responses to the synthetic polypeptide (T,G)-A- -L; those which did not make $T$ helper factor (an antigen-specific mediator of $T$ cell help) and which was interpreted as a $\mathrm{T}$ cell defect, and those which did not respond to $\mathrm{T}$ cell helper factor, which was interpreted as a B cell defect. But since macrophages are needed for both these reactions a macrophage defect could be at the root of both these failures. $\mathrm{T}$ cell suppression has also been proposed as a mechanism of non-responsiveness and since suppression is much less dependent on macrophage function than $T$ cell help, a net suppression would occur in the presence of dysfunctional macrophages. This idea is not incompatible with the proposed role of the macrophage.

Whatever the truth of the mechanism of Ir gene action, it now seems certain that macrophages and macrophage-like cells have a more complex role in the immune system than hitherto suspected, and the precise nature of the antigen-presenting cell needs to be re-examined. Are these classical macrophage or lymphocyte-like cells, or a hybrid between the two. In view of the plethora of lymphocyte subpopulations recognised in the past two years, the existence of yet another type of cell in the immune system need not be too improbable. However, the question still remains as to whether there are Ir genes expressed by other cells of the immune system.

turb the medium it purports to measure. Small barium releases, such as that used by Deehr and Romick, do not distort the electric fields seriously although they must have some effect. Their observation is not the first of perturbations in the ionosphere beneath a barium cloud. Stoffregen (J. atmos. terrestr. Phys. 32, 171; 1970) detected a temporary increase in the auroral line at $557.7 \mathrm{~nm}$ at $100 \mathrm{~km}$ altitude below a barium cloud. He did not observe pulsations and there was apparently little natural aurora present. Spracklen and Jones $(Q . J l R$. Astr. 